\title{
NOTUM is a potential pharmacodynamic biomarker of Wnt pathway inhibition
}

\author{
Babita Madan ${ }^{1}$, Zhiyuan Ke ${ }^{2}$, Zheng Deng Lei ${ }^{1}$, Frois Ashley Oliver $^{1}$, Masanobu \\ Oshima ${ }^{3}$, May Ann Lee ${ }^{2}$, Steve Rozen ${ }^{1}$, David M. Virshup ${ }^{1,4}$ \\ ${ }^{1}$ Program in Cancer and Stem Cell Biology, Duke-NUS Medical School, Singapore \\ ${ }^{2}$ Experimental Therapeutics Centre, A*STAR, Biopolis, Singapore \\ ${ }^{3}$ Division of Genetics, Cancer Research Institute, Kanazawa University, Kanazawa, Japan \\ ${ }^{4}$ Department of Pediatrics, Duke University, Durham, NC, USA \\ Correspondence to: David M. Virshup, e-mail: David.Virshup@duke-nus.edu.sg \\ Babita Madan, e-mail: Babita.Madan@duke-nus.edu.sg \\ Keywords: Wnt signaling, Notum, Biomarker, PORCN inhibitor \\ Received: September 06, $2015 \quad$ Accepted: January 23, $2016 \quad$ Published: February 03, 2016
}

\begin{abstract}
Activation of Wnt signaling due to Wnt overexpression or mutations of Wnt pathway components is associated with various cancers. Blocking Wnt secretion by inhibiting PORCN enzymatic activity has shown efficacy in a subset of cancers with elevated Wnt signaling. Predicting response to upstream Wnt inhibitors and monitoring response to therapeutics is challenging due to the paucity of well-defined biomarkers. In this study we identify Notum as a potential biomarker for Wnt driven cancers and show that coordinate regulation of NOTUM and AXIN2 expression may be a useful predictor of response to PORCN inhibitors. Most importantly, as NOTUM is a secreted protein and its levels in blood correlate with tumor growth, it has potential as a pharmacodynamic biomarker for PORCN and other Wnt pathway inhibitors.
\end{abstract}

\section{INTRODUCTION}

Wnts are secreted morphogens essential for embryonic development and tissue homeostasis $[1,2]$. Dysregulated Wnt signaling causes various developmental abnormalities and diseases such as cancers, fibrosis and osteoporosis [3]. Interaction of Wnts with the cell surface receptors Frizzled, low-density lipoprotein receptorrelated proteins 5 and 6 (LRP 5/6) and G protein coupled receptors activates both $\beta$-catenin dependent and $\beta$-catenin independent pathways. The mono-unsaturated palmitoleic acid conjugated to a serine residue conserved in all the Wnts is essential for the engagement of Wnts with its receptors Frizzleds as the palmitoleate group inserts into a hydrophobic groove of the Fzd cysteine rich domain [4]. This palmitoleation of Wnts mediated by an ER resident enzyme Porcupine (PORCN) is also essential for their secretion. Mutating the conserved serine residue in Wnt, or inhibition of PORCN enzymatic activity prevents the interaction of Wnts with their carrier protein Wntless (WLS) and hence blocks Wnt secretion [4-6].

$\mathrm{Wnt} / \beta$-catenin signaling regulates expression of its own regulators, including the negative regulators of $\mathrm{Wnt} / \beta$ - catenin signaling, AXIN2 and NOTUM. NOTUM encodes a secreted Wnt antagonist that regulates WNT/Wg activity gradients in Drosophila imaginal discs and in vertebrate embryogenesis [7-9]. While originally proposed to cleave glycosylphosphatidylinositol linkages, more recently NOTUM has been shown to be a carboxyl oxoesterase that functions as a Wnt antagonist by deacylating Wnts $[8,10]$. The crystal structure demonstrates that NOTUM has a large hydrophobic pocket that can accommodate cis-unsaturated fatty acid palmitoleate [10]. The glycosaminoglycan binding sites on NOTUM facilitate its interaction with heparin sulphate glycoproteins, glypicans that act as scaffolds to co-localize NOTUM with its substrate Wnt proteins.

Preventing the secretion of Wnts by inhibiting the PORCN enzymatic activity inhibits both canonical and non-canonical Wnt signaling; hence PORCN inhibitors have shown high efficacy in the subset of cancers driven by high Wnt signaling including molecularly defined colorectal cancers with $\mathrm{R}$-spondin translocations and pancreatic cancers with RNF43 mutations [11]. As these inhibitors advance to the clinical trials, identification of predictive and pharmacodynamic markers is imperative 
for the selection and treatment of patients. In this study we show that regulation of NOTUM expression correlates with sensitivity to PORCN inhibitors. We also show that NOTUM can be a potential pharmacodynamic biomarker for Wnt pathway inhibitors.

\section{RESULTS AND DISCUSSION}

\section{NOTUM is a pharmacodynamic biomarker for PORCN inhibitors in vitro}

AXIN2 expression has been used extensively as readout for Wnt pathway activity. To identify cancers sensitive to Wnt secretion inhibitors, 13 pancreatic cell lines were treated with 2 structurally unrelated porcupine inhibitors Wnt-C59 $\left(\mathrm{IC}_{50}=0.1 \mathrm{nM}\right)$ and ETC-159 $\left(\mathrm{IC}_{50}=3\right.$ $\mathrm{nM})[6,11]$ for $24 \mathrm{~h}$. In 9 cell lines, the PORCN inhibitors reduced AXIN2 mRNA expression by more than 50\%, suggesting the presence of endogenous autocrine Wnt signaling (Figure 1A). We next tested if this Wnt autocrine signaling was important for growth of these 9 cell lines in which PORCN inhibition reduced AXIN2 expression. We tested the effect of ETC-159 on their proliferation after low-density plating. However, drug-induced downregulation of AXIN2 expression did not correlate well with drug-induced inhibition of proliferation. The proliferation of 4 cell lines was inhibited, while 5 cell lines were not affected even in high concentration $(1 \mu \mathrm{M})$ of ETC-159 (Figure 1B).

As the drug-dependent repression of AXIN2 gene expression correlated poorly with inhibition of proliferation after low density plating, we investigated additional Wnt/ $\beta$-catenin target genes that could serve as predictive or pharmacodynamic biomarkers. Analysis of the Singapore and Australian gastric cancer tissue databases for expression of $\beta$-catenin target genes revealed that NOTUM mRNA expression highly correlated (correlation $\geq 0.64$ ) with AXIN2 mRNA expression (Table 1A-1B). Consistent with this, Wnt-driven gastric tumors from K19-Wnt1/ $C 2 m E$ mice [14] that overexpress Wnt1, Ptgs 2 and Ptges in the gastric epithelium, also had high Notum mRNA expression (Figure 1C). NOTUM is a Wnt/ $\beta$-catenin target gene [15] reported to negatively regulate Wnt signaling in zebrafish [7] and is potentially valuable as a biomarker because it is a secreted protein. We confirmed that ectopic expression of human NOTUM inhibits signaling activity driven by diverse Wnts (Figure 1D).

To determine if NOTUM expression was indeed regulated by Wnt signaling, we measured the abundance of NOTUM mRNA in the same 13 cell lines following PORCN inhibition (Figure 2A). NOTUM transcript expression was suppressed by PORCN inhibition in a subset of the cells. Notably, only the cell lines in which both NOTUM and AXIN2 were suppressed by more than $50 \%$ by PORCN inhibition were growth-inhibited by ETC159 (Figure 1B). Downregulation of NOTUM expression strongly associates with response to PORCN inhibitor, $\mathrm{p}$ value $=0.0028$ (Wilcoxon rank sum test). Of note, 3 of these 4 cell lines have loss of function mutations in RNF43, which sensitizes cells to Wnts $[11,16]$. Notably not all cell lines with RNF43 mutation such as Panc10.05 (M18fs) were sensitive to PORCN inhibition despite decreased AXIN2 expression. These data suggest that coordinated reduction of AXIN2 and NOTUM mRNA expression may be a better predictor of Wnt addiction and response to PORCN inhibitors than each gene individually.

\section{Notum is a pharmacodynamic biomarker for PORCN inhibitors in vivo}

We identified NOTUM gene expression as a good predictor for response to PORCN inhibitors in pancreatic cell lines in vitro. To test if its expression also correlated with the inhibition of Wnt signaling pathway in tumors in vivo, we evaluated Notum gene expression in Wnt dependent cancer models. Mice carrying an MMTV LTRWntl transgene have marked overexpression of Wntl in the mammary gland, driving hyperplasia and an eventual development of adenocarcinomas [17]. Tumor fragments from MMTV-Wnt1 tumors were orthotopically transplanted into the $4^{\text {th }}$ mammary fat pad of BALB/c nude mice. We have previously reported that once daily gavage dosing of ETC-159 significantly inhibited the growth of these tumors [11]. NOTUM was high in the tumors from the vehicletreated mice and was decreased significantly in the ETC159 treated mice (Figure 2B). We then tested a second Wnt-dependent human pancreatic cell line. We and others have shown that PORCN inhibition effectively prevents the growth of HPAF-II tumors, a pancreatic cancer cell line with an RNF43 mutation $[11,16]$. A remarkable decrease in NOTUM expression was observed 56 hours after the start of ETC-159 treatment of established HPAF-II orthotopic pancreatic xenografts (Figure 2B). Taken together our data demonstrate that NOTUM expression is a useful predictor of response to PORCN inhibitors in vivo.

NOTUM is a secreted protein and therefore has the potential to be used as pharmacodynamic biomarker to monitor therapeutic response. We analyzed the protein levels of NOTUM in the plasma from the MMTV-Wnt1 mice pre-and post-PORCN inhibitor treatment (Figure 2C $\& 2 \mathrm{D})$. Basal levels of NOTUM in the BALB/c nude mice and the MMTV-Wnt1 transgenic mice were low. NOTUM protein abundance in the plasma increased when the mice develop tumors spontaneously (MMTV-Wnt1 transgenic mice, Figure 2D) or when tumors are established in BALB/c nude mice (Figure 2D). Following treatment with two independent PORCN inhibitors, ETC-159 (BALB/c nude mice, Figure 2C) or Wnt-C59 (MMTV-Wnt1 transgenic, Figure 2D) the levels of NOTUM in the blood decreased considerably to those seen in the control mice. These results demonstrate that NOTUM may be a useful pharmacodynamic marker for Wnt pathway inhibitors. 
A

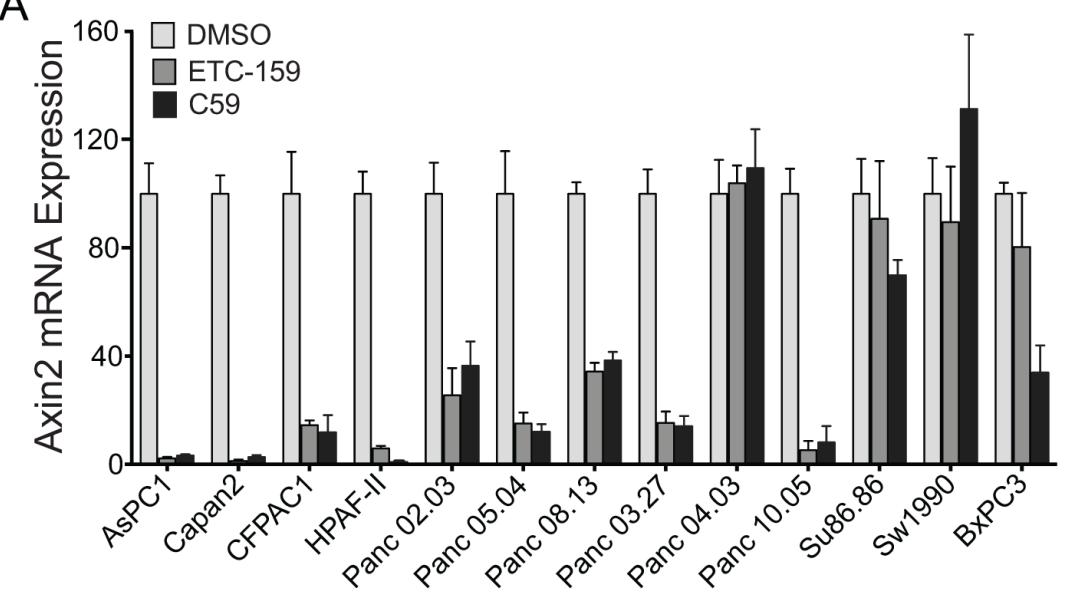

$\mathrm{B}$

DMSO ETC-159 (1 uM)

DMSO ETC-159 (1 uM)
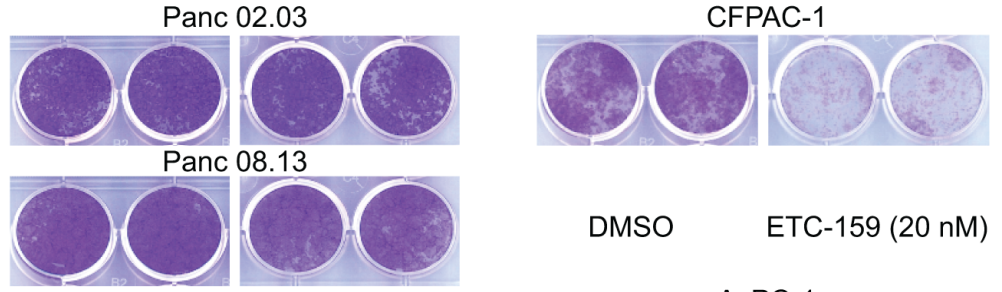

DMSO

ETC-159 (20 nM)
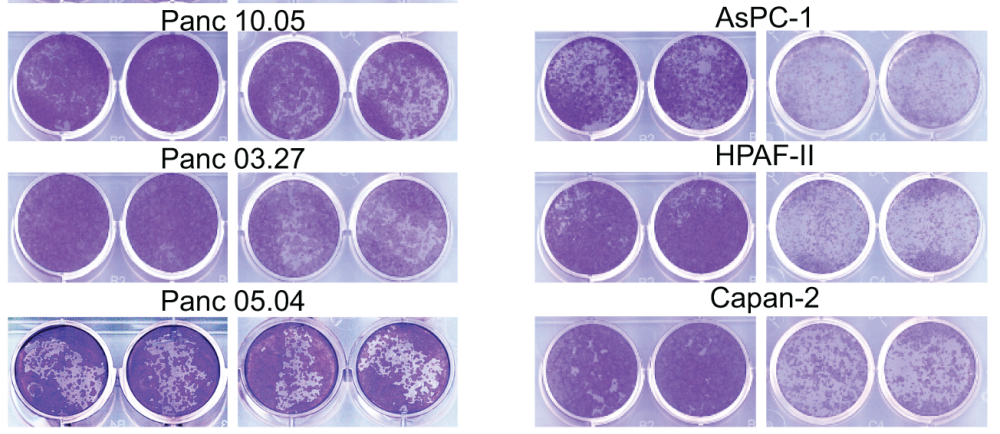

Capan-2

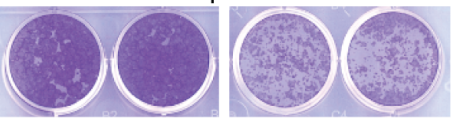

C
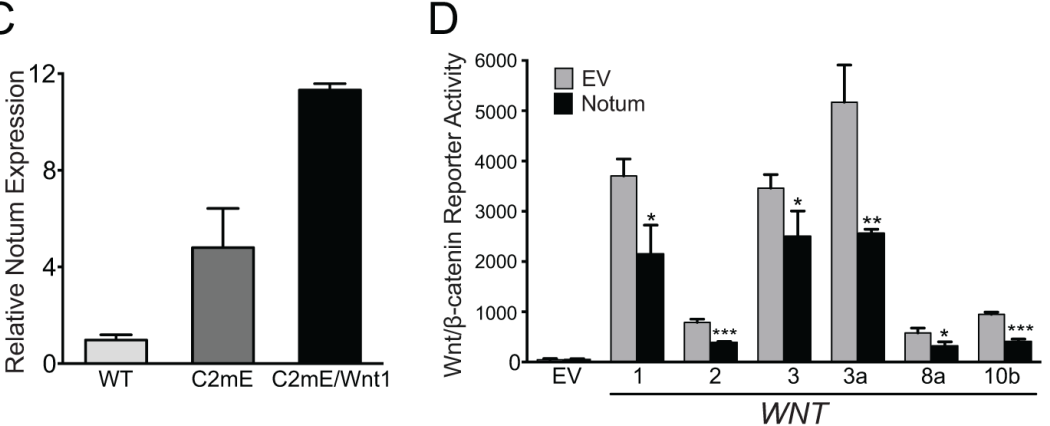

Figure 1: AXIN2 gene expression is a poor predictor of PORCN inhibitor sensitivity. A. AXIN2 expression is widely suppressed in pancreatic cell lines treated with Wnt-C59 and ETC-159: Pancreatic cancer cell lines were treated with $100 \mathrm{nM}$ ETC-159 or $100 \mathrm{nM}$ Wnt-C59 for 24h. Total RNA was isolated and AXIN2 expression was analyzed by qRT-PCR. Expression of AXIN2 normalized to 18SrRNA in the ETC-159 or Wnt-C59 treated cells is represented as the percentage of respective DMSO treated controls. B. ETC-159 blocks foci formation in a subset of pancreatic cell lines: 1000-3000 cells of the indicated pancreatic cell lines were plated in 24 well plates and treated with ETC-159 $(10 \mathrm{nM}$ to $1 \mu \mathrm{M})$. Cell number and the duration for treatment were determined based on the growth rate of each line. Following methanol fixation the cells were stained with crystal violet. C. Expression of Notum is elevated in Gan mice: Total RNA was isolated from gastric tissue of K19-C2mE (C2mE) and K19-C2mE/Wnt1 (Gan) mice and Notum expression was measured by qRT-PCR. Expression was normalized to Hprt, $\mathrm{n}=5$ mice /group. D. NOTUM inhibits Wnt/B-catenin reporter activity induced by diverse Wnts: HT1080 cells were transiently transfected with the Super8xTOPFLASH (STF), NOTUM and the indicated Wnt expression plasmids. The reporter activity was normalized to mCherry expression, a control for transfection efficiency. Bars represent the mean \pm SD. $* * * p \leq 0.001, * * p \leq 0.01, * p \leq 0.05$. 
Table 1: $A X I N 2$ correlated genes

(A) Singapore Gastric cancer dataset

\begin{tabular}{|c|c|c|c|c|}
\hline Probeset & Correlation & P value & FDR & Gene \\
\hline 224176_s_at & 0.92 & $\mathbf{0}$ & $\mathbf{0}$ & AXIN2 \\
\hline 222696_at & 0.86 & $\mathbf{0}$ & $\mathbf{0}$ & AXIN2 \\
\hline 222695_s_at & 0.74 & $\mathbf{0}$ & $\mathbf{0}$ & AXIN2 \\
\hline 228649 at & 0.64 & $\mathbf{0}$ & $\mathbf{0}$ & NOTUM \\
\hline 229481_at & 0.63 & 0 & 0 & LOC283859 \\
\hline 224498_x_at & 0.62 & $\mathbf{0}$ & $\mathbf{0}$ & AXIN2 \\
\hline 235845_at & 0.59 & 0 & 0 & SP5 \\
\hline 207607_at & 0.59 & 0 & 0 & ASCL2 \\
\hline 209494_s_at & 0.56 & 0 & 0 & PATZ1 \\
\hline 226360_at & 0.55 & 0 & 0 & ZNRF3 \\
\hline 229215_at & 0.54 & $2.22 \mathrm{E}-16$ & $1.01 \mathrm{E}-12$ & ASCL2 \\
\hline 218704_at & 0.53 & $4.44 \mathrm{E}-16$ & $1.87 \mathrm{E}-12$ & RNF43 \\
\hline 208608_s_at & 0.52 & $2.89 \mathrm{E}-15$ & $1.13 \mathrm{E}-11$ & SNTB1 \\
\hline 240211_at & 0.51 & $6.22 \mathrm{E}-15$ & $2.26 \mathrm{E}-11$ & LOC100130468 \\
\hline
\end{tabular}

(B) Australian Gastric cancer dataset

\begin{tabular}{|c|c|c|c|c|}
\hline Probeset & Correlation & P value & FDR & Gene \\
\hline 224176_s_at & 0.93 & 0 & 0 & AXIN2 \\
\hline 222696_at & 0.88 & 0 & 0 & AXIN2 \\
\hline 229481_at & 0.69 & $3.36 \mathrm{E}-11$ & $6.12 \mathrm{E}-07$ & LOC283859 \\
\hline 222695_s_at & 0.68 & $6.73 \mathrm{E}-11$ & $9.19 \mathrm{E}-07$ & AXIN2 \\
\hline 205983_at & 0.66 & $4.18 \mathrm{E}-10$ & $4.57 \mathrm{E}-06$ & DPEP1 \\
\hline 1555497_a_at & 0.66 & $6.65 \mathrm{E}-10$ & $6.05 \mathrm{E}-06$ & CYP4B1 \\
\hline 208121_s_at & 0.65 & $1.14 \mathrm{E}-09$ & $8.89 \mathrm{E}-06$ & PTPRO \\
\hline 228649_at & 0.65 & $1.42 E-09$ & 9.69E-06 & NOTUM \\
\hline 210550_s_at & 0.64 & $1.88 \mathrm{E}-09$ & $1.14 \mathrm{E}-05$ & RASGRF1 \\
\hline 231814_at & 0.62 & $9.04 \mathrm{E}-09$ & $4.75 \mathrm{E}-05$ & MUC12 \\
\hline 218704_at & 0.62 & $1.14 \mathrm{E}-08$ & $5.17 \mathrm{E}-05$ & RNF43 \\
\hline 205555 s_at & 0.61 & $1.43 \mathrm{E}-08$ & $6.03 \mathrm{E}-05$ & MSX2 \\
\hline
\end{tabular}

A subset of cancers are dependent on Wnt signaling, and can in part be identified by the presence of mutations that increase cellular sensitivity to Wnts [18]. New therapies that target Wnt signaling show activity in genetically identified Wnt-addicted cancers. One key feature of the response to these new therapies is differentiation, rather than apoptosis. This may account for the slow regression of these tumors in pre-clinical models $[11,16,19]$. It would be helpful to have more rapid readouts of tumor response. Measurement of changes in secreted proteins is one approach that is useful in other settings [20]. Here we demonstrate that PORCN inhibitors inhibit the Wnt target gene NOTUM. In addition, NOTUM protein can be detected in the blood of mice bearing Wnt-dependent MMTV-Wnt1 tumors and its abundance drops significantly upon therapy. These studies suggest that it would be useful to develop highly sensitive assays for circulating NOTUM protein and assess their utility in patients with Wnt-driven cancers. 
A

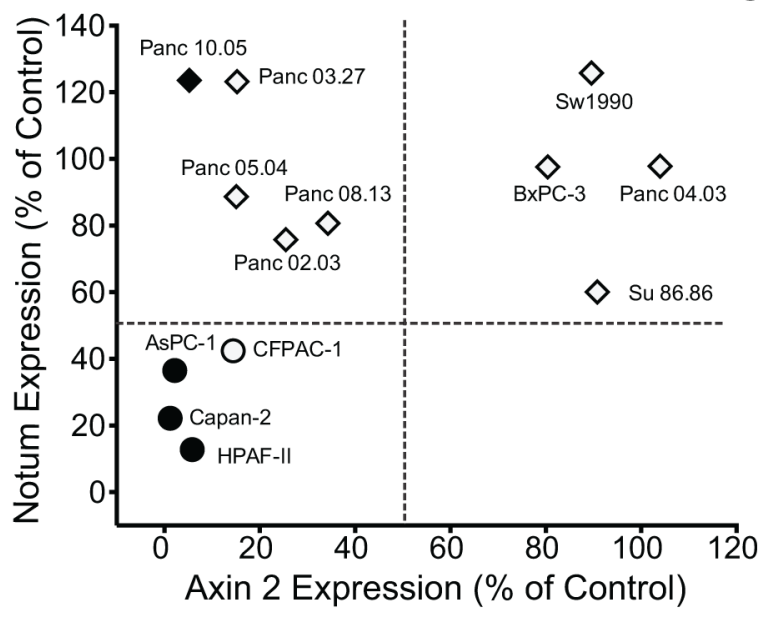

C

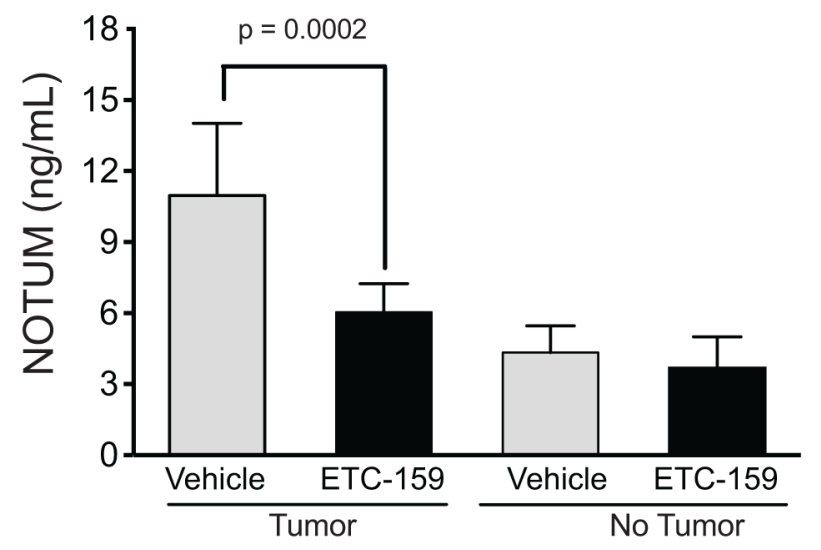

B
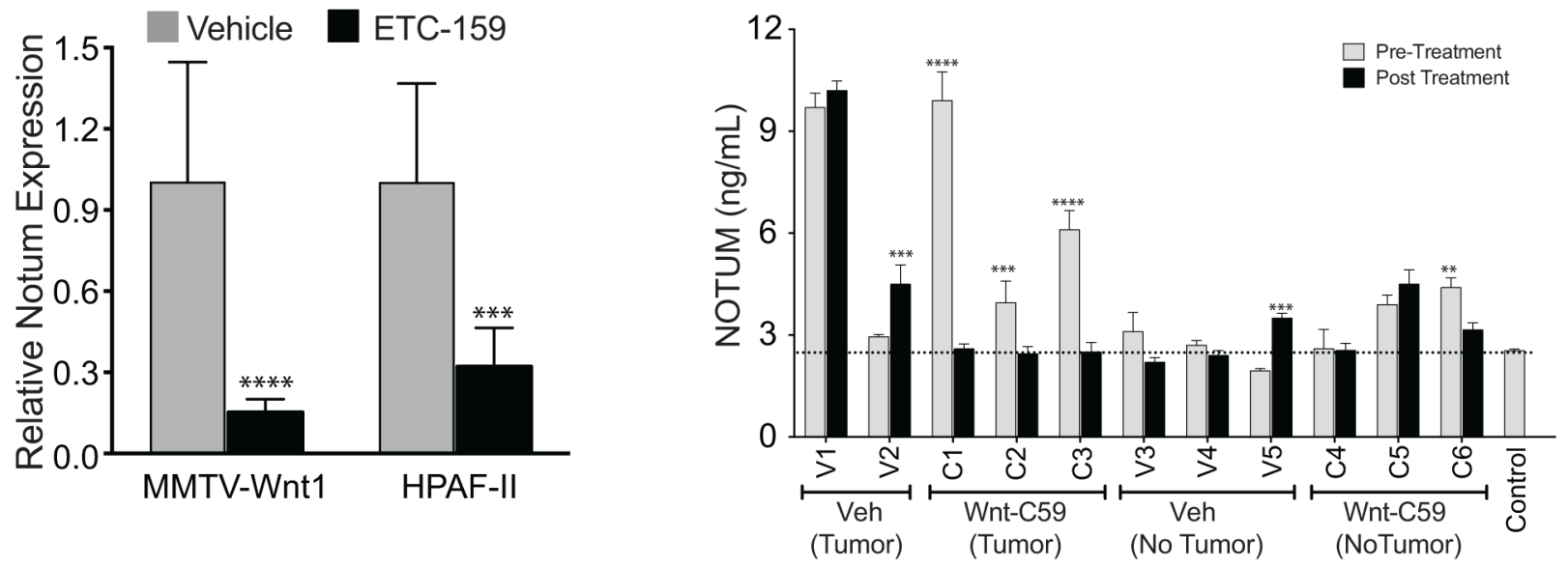

Figure 2: A. Combined inhibition of NOTUM and AXIN2 expression predicts sensitivity to PORCN inhibitors: $A X I N 2$ and NOTUM expression was assessed in cell lines exposed to $100 \mathrm{nM}$ ETC-159 for 24 hours. RNF43 mutant (filled symbols) and wild-type (open symbols) cell lines. ETC-159 sensitive (circle) or insensitive (diamond) cell lines. B. ETC-159 treatment decreases Notum expression in MMTV-Wnt1 allografts and HPAF-II tumors: RNA isolated from vehicle or ETC-159 treated MMTV-Wnt1 tumors $(\mathrm{n}=9$ in each group) or HPAF-II tumors ( $\mathrm{n}=6$ in each group) was assessed by RT-qPCR. Data presented are mean \pm SD, Data were analyzed using unpaired t-test, $* * * * p \leq 0.0001, * * * p \leq 0.001$. C. NOTUM protein is decreased in plasma of ETC-159 treated mice: Control BALB/c nude mice or mice with established MMTV-Wnt1 tumors were treated daily for 10 days with ETC-159 (30 mg/kg). Blood was collected 6 hours after the last dose and plasma NOTUM levels were measured using ELISA. The graph shows group means $\pm \mathrm{SD} . \mathrm{n}=10$ for tumor bearing mice in each group and $\mathrm{n}=4$ for control BALB/c nude mice. D. Wnt-C59 treatment decreases NOTUM protein levels in MMTV-Wnt1 mice: Plasma NOTUM levels were measured in control (C57/B16, n=4) or transgenic MMTV-Wnt1 mice before initiating the treatment and after 3 days of treatment with $10 \mathrm{mg} / \mathrm{kg}$ Wnt-C59 as measured by ELISA. Data represents plasma NOTUM levels of individual mice. Dotted line represents NOTUM levels in control mice. V=vehicle-treated mice, $\mathrm{C}=\mathrm{Wnt}-\mathrm{C} 59$ treated mice. $* * * \mathrm{p} \leq 0.001, * * * * \mathrm{p} \leq 0.0001$.

\section{MATERIALS AND METHODS}

\section{RNA isolation and qRT-PCR}

All the cell lines were obtained from ATCC and were tested free of mycoplasma contamination. Total RNA was isolated from the cell lines or tumors using RNAeasy kit (Qiagen). RNA was reverse transcribed with iScript reverse transcriptase (BioRAD) and real time quantitative PCR (qPCR) was performed with SsoFast ${ }^{\mathrm{TM}}$ EvaGreen ${ }^{\circledR}$ assay Supermix from BioRad. HPRT was used as housekeeping gene. The primers used are Axin2 F: 5'-CTCCCCACCTTGAATGAAGA-3', R: 5'-TGGCTGGTGCAAAGACATAG-3'; Hprt F: 5'-CCT CACTGCTTTCCGGAGCGG -3' R: 5'-ATCGCTAATCA CGACGCTGGGA-3'; Notum F: 5'- GGAAGGCCA GTG GCTATACATC-3', 5'- GTCCGTCCAATAGCT CC GTATG-3'. 


\section{TOPFLASH assays}

HT1080 cells were transfected in 24-well plates with $50 \mathrm{ng}$ Wnt, $100 \mathrm{ng}$ mCherry, without or with Notum and $550 \mathrm{ng}$ Super 8x TOPFLASH plasmids (a gift from Randall $\mathrm{T}$ Moon). $24 \mathrm{~h}$ after transfection, the cells were washed with PBS and lysed in $0.6 \%$ NP40 in PBS containing protease inhibitors. Super8xTOPFLASH reporter activity was measured using firefly luciferase substrate (Promega, Madison WI). The reporter activity was normalized to the transfection efficiency as determined using mCherry expression [12].

\section{Animal care}

MMTV-Wnt1 mice were obtained from Jackson Laboratories (Bar Harbor, Maine) and were maintained by backcrossing to $\mathrm{C} 57 \mathrm{BL} / 6$. BALB/c nude mice were purchased from InVivos, Singapore. The Duke-NUS Institutional Animal Care and Use Committee approved all the animal studies for compliance with regulations. Animals were housed in standard cages and were allowed access ad libitum to food and water.

\section{Tumor implantation, treatment of mice and analysis of plasma}

Mouse xenograft models were established by subcutaneous implantation of MMTV-Wnt1-derived solid tissue fragments in BALB/c nude mice. Following development of palpable tumors, treatment was initiated. Mice were treated with ETC-159 formulated in 50\% PEG400 (vol/vol) in water and administered by oral gavage at a dosing volume of $10 \mu \mathrm{L} / \mathrm{g}$ body weight. At sacrifice, blood was collected and plasma was isolated. Notum levels in the plasma were measured using ELISA kit from CusABio (CSB-EL015955HU) following manufacturer's protocol.

\section{Microarray data analysis}

To identify correlation between AXIN2 and other genes, expression data from 201 gastric tumors from Singapore (GSE15459 and GSE34942) and 70 gastric tumors from Australia (GSE35809) was analyzed [13]. AXIN2 expression was calculated as an average expression value of its corresponding probesets (222695_s_at, 222696_at, 224176_s_at, and 224498_x_at). Pearson correlation coefficient was calculated for each probeset of the gene against the average expression level of AXIN2. p-values were adjusted to account for multiple testing using the Benjamini-Hochberg (FDR) correction.

\section{Data analysis}

Data was analyzed using Prism v5.0 (GraphPad). Significance for all tests was set at $\mathrm{p} \leq 0.05$ unless otherwise stated.

\section{ACKNOWLEGMENTS}

We acknowledge the technical assistance of members of the Virshup lab and members of Experimental Therapeutics Centre.

This research is supported in part by the National Research Foundation Singapore and administered by the Singapore Ministry of Health's National Medical Research Council under the STAR Award Program to D.M.V.. The research in Experimental Therapeutics Centre is supported by the Agency for Science, Technology and Research (A*STAR), Singapore.

\section{CONFLICTS OF INTEREST}

Babita Madan, David M. Virshup, May Ann Lee and Zhiyuan Ke are co-inventors of a small molecule PORCN inhibitor.

\section{REFERENCES}

1. Chien AJ, Conrad WH, Moon RT. A Wnt Survival Guide: From Flies to Human Disease. Journal of Investigative Dermatology. 2009; 129:1614-27.

2. Varmus H, Nusse R. Three decades of Wnts: a personal perspective on how a scientific field developed. EMBO J. 2012; 31:2670-84.

3. Yu J, Virshup DM. Updating the Wnt pathways. Biosci Rep. 2014; 34.

4. Janda CY, Waghray D, Levin AM, Thomas C, Garcia KC. Structural Basis of Wnt Recognition by Frizzled. Science. 2012; 337:59-64.

5. Coombs GS, Yu J, Canning CA, Veltri CA, Covey TM, Cheong JK, Utomo V, Banerjee N, Zhang ZH, Jadulco RC, Concepcion GP, Bugni TS, Harper MK, Mihaleck I, et al. WLS-dependent secretion of WNT3A requires Ser209 acylation and vacuolar acidification. Journal of Cell Science. 2010; 123:3357-67.

6. Proffitt KD, Madan B, Ke Z, Pendharkar V, Ding L, Lee MA, Hannoush RN, Virshup DM. Pharmacological Inhibition of the Wnt Acyltransferase PORCN Prevents Growth of WNT-Driven Mammary Cancer. Cancer Research. 2013; 73:502-7.

7. Flowers GP, Topczewska JM, Topczewski J. A zebrafish Notum homolog specifically blocks the Wnt/ $\beta$-catenin signaling pathway. Development. 2012; 139:2416-25.

8. Zhang X, Cheong S-M, Amado NG, Reis AH, MacDonald BT, Zebisch M, Jones Y, Abreu JG, He X. Notum Is Required for Neural and Head Induction via Wnt Deacylation, Oxidation, and Inactivation. Developmental Cell. 2015; 32:719-30.

9. Giráldez AJ, Copley RR, Cohen SM. HSPG modification by the secreted enzyme Notum shapes the Wingless morphogen gradient. Developmental Cell. 2002; 2:667-76. 
10. Kakugawa S, Langton PF, Zebisch M, Howell SA, Chang T-H, Liu Y, Feizi T, Bineva G, O’Reilly N, Snijders AP, Jones EY, Vincent JP Notum deacylates Wnt proteins to suppress signalling activity. Nature. 2015; 519:187-92.

11. Madan B, Ke Z, Harmston N, Yei HS, Frois AO, Alam J, Jeyaraj DA, Pedharkar V, Ghosh K, Virshup IH, Manoharan V, Ong EHQ Sangthongpitag K, et al. Wnt addiction of genetically defined cancers reversed by PORCN inhibition. Oncogene. 2015; 1-33.

12. Coombs GS, Schmitt AA, Canning CA, Alok A, Low ICC, Banerjee N, Kaur S, Utomo V, Jones CM, Pervaiz S, Toone EJ, Virshup DM. Modulation of Wnt/ $\beta$-catenin signaling and proliferation by a ferrous iron chelator with therapeutic efficacy in genetically engineered mouse models of cancer. Oncogene. 2011; 31:213-25.

13. Lei Z, Tan IB, Das K, Deng N, Zouridis H, Pattison S, Chua C, Feng Z, Guan YK, Ooi CH, Ivanova T, Zhang S, Lee M et al. Identification of molecular subtypes of gastric cancer with different responses to PI3-kinase inhibitors and 5-fluorouracil. Gastroenterology. 2013; 145:554-65.

14. Oshima H, Oshima M. The inflammatory network in the gastrointestinal tumor microenvironment: lessons from mouse models. J Gastroenterol. 2012; 47:97-106.

15. Torisu Y, Watanabe A, Nonaka A, Midorikawa Y, Makuuchi M, Shimamura T, et al. Human homolog of NOTUM, overexpressed in hepatocellular carcinoma, is regulated transcriptionally by $\beta$-catenin/TCF. Cancer Sci. 2008; 99:1139-46.

16. Jiang X, Hao HX, Growney JD, Woolfenden S, Bottiglio C, Ng N, Lu B, Hsieh MH, Bagdasarian L, Meyer R, Smith TR, Avello M, et al. Inactivating mutations of RNF43 confer Wnt dependency in pancreatic ductal adenocarcinoma. Proc Natl Acad Sci USA. 2013; 110:12649-54.

17. Tsukamoto AS, Grosschedl R, Guzman RC, Parslow T, Varmus HE. Expression of the int-1 gene in transgenic mice is associated with mammary gland hyperplasia and adenocarcinomas in male and female mice. Cell. 1988; 55:619-25.

18. Madan B, Virshup DM. Targeting Wnts at the Source-New Mechanisms, New Biomarkers, New Drugs. Molecular Cancer Therapeutics. 2015; 14:1087-94.

19. Gurney A, Axelrod F, Bond CJ, Cain J, Chartier C, Donigan J, Fisher M, Chaudhari A, Ji M, Kapoun AM, Lam A, Lazetic S, Ma S, et al. Wnt pathway inhibition via the targeting of Frizzled receptors results in decreased growth and tumorigenicity of human tumors. Proc Natl Acad Sci USA. 2012; 109:11717-22.

20. Pommier AJC, Shaw R, Spencer SKM, Morgan SR, Hoff PM, Robertson JD, Barry ST, Jüngenssmeier JM. Serum protein profiling reveals baseline and pharmacodynamic biomarker signatures associated with clinical outcome in mCRC patients treated with chemotherapy \pm cediranib. $\mathrm{Br}$ J Cancer. 2014; 111:1590-604. 